This item was submitted to Loughborough's Research Repository by the author.

Items in Figshare are protected by copyright, with all rights reserved, unless otherwise indicated.

\title{
Coarsening modes of clusters of aggregating particles
}

PLEASE CITE THE PUBLISHED VERSION

http://dx.doi.org/10.1103/PhysRevE.89.032144

\section{PUBLISHER}

(C) American Physical Society

VERSION

VoR (Version of Record)

LICENCE

CC BY-NC-ND 4.0

\section{REPOSITORY RECORD}

Pototsky, Andrey, Uwe Thiele, and Andrew J. Archer. 2019. "Coarsening Modes of Clusters of Aggregating Particles". figshare. https://hdl.handle.net/2134/15354. 
This item was submitted to Loughborough's Institutional Repository (https://dspace.lboro.ac.uk/) by the author and is made available under the following Creative Commons Licence conditions.

\section{creative
commons}

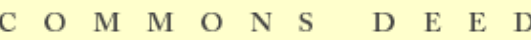

Attribution-NonCommercial-NoDerivs 2.5

You are free:

- to copy, distribute, display, and perform the work

Under the following conditions:

Attribution. You must attribute the work in the manner specified b the author or licensor.

Noncommercial. You may not use this work for commercial purposes.

No Derivative Works. You may not alter, transform, or build upon this work.

- For any reuse or distribution, you must make clear to others the license terms of this work.

- Any of these conditions can be waived if you get permission from the copyright holder.

Your fair use and other rights are in no way affected by the above.

This is a human-readable summary of the Leqal Code (the full license).

\section{Disclaimer 만}

For the full text of this licence, please go to: http://creativecommons.org/licenses/by-nc-nd/2.5/ 


\title{
gु \\ Coarsening modes of clusters of aggregating particles
}

\author{
Andrey Pototsky, ${ }^{1}$ Uwe Thiele, ${ }^{2,3}$ and Andrew J. Archer ${ }^{2}$ \\ ${ }^{1}$ Department of Mathematics, Faculty of Science, Engineering and Technology, Swinburne University of Technology, \\ Hawthorn, Victoria, 3122, Australia \\ ${ }^{2}$ Department of Mathematical Science, Loughborough University, Loughborough LE11 3TU, United Kingdom \\ ${ }^{3}$ Institut für Theoretische Physik, Westfälische Wilhelms-Universität Münster, Wilhelm Klemm Strasse 9, D-48149 Münster, Germany
}

(Received 27 January 2014; published 31 March 2014)

\begin{abstract}
There are two modes by which clusters of aggregating particles can coalesce: The clusters can merge either (i) by the Ostwald ripening process, in which particles diffuse from one cluster to the other while the cluster centers remain stationary, or (ii) by means of a cluster translation mode, in which the clusters move toward each other and join. To understand in detail the interplay between these different modes, we study a model system of hard particles with an additional attraction between them. The particles diffuse along narrow channels with smooth or periodically corrugated walls, so that the system may be treated as one-dimensional. When the attraction between the particles is strong enough, they aggregate to form clusters. The channel potential influences whether clusters can move easily or not through the system and can prevent cluster motion. We use dynamical density functional theory to study the dynamics of the aggregation process, focusing in particular on the coalescence of two equal-sized clusters. As long as the particle hard-core diameter is nonzero, we find that the coalescence process can be halted by a sufficiently strong corrugation potential. The period of the potential determines the size of the final stable clusters. For the case of smooth channel walls, we demonstrate that there is a crossover in the dominance of the two different coarsening modes, which depends on the strength of the attraction between particles, the cluster sizes, and the separation distance between clusters.
\end{abstract}

DOI: 10.1103/PhysRevE.89.032144

\section{INTRODUCTION}

The late-stage dynamics of phase separation is often characterized by the presence of droplets, islands, or clusters of one phase dispersed in a surrounding medium of another phase. This may, for example, be droplets of a liquid condensing from a supersaturated vapor, phase separation in a binary mixture or alloy, or the condensation to form islands of a liquid or solid phase as particles diffuse over a surface. Over time, the average size of the clusters or droplets grows and the total number decreases. There are two mechanisms by which this can occur. The first is often referred to as Ostwald ripening, in which particles diffuse from the smaller clusters to the larger clusters through the surrounding phase while the centers of the clusters remain fixed in space. Lifshitz and Slyozov [1] and Wagner [2] were the first to develop a theory for this process. For a more recent discussion, see, for example, Refs. [3-8]. The second mechanism by which the clusters may coarsen is via the motion of entire clusters toward other clusters and subsequently joining. This motion may simply be via droplet diffusion [3]. Alternatively, it can be due to an imposed external driving, e.g., by gravity in sedimentation, creaming, and the formation of rain drops. Quite often, cluster diffusion may be neglected compared to the diffusion of individual particles since the diffusion coefficient $D$ through the surrounding material decreases with the inverse of the radius $R$ of the diffusing object, e.g., in three dimensions, $D \sim 1 / R$.

Since the radius of a cluster is much larger than an individual particle, the cluster diffusion coefficient is much smaller. However, this is not always the case, particularly when two drops come close enough to each other that they begin to interact strongly. Then capillary and other such forces can lead to their motion and may result in cluster-cluster coalescence.

There are other aspects and theories of aggregation kinetics that are worth mentioning: In earlier models, the
PACS number(s): 05.40.-a, 05.60.-k, 68.43.Jk, 87.15.nr

aggregation process was regarded as irreversible, whereby the thermally activated escape of single particles from clusters was neglected. In this regime, growth occurs solely due to collisions between smaller clusters that encounter each other traveling by diffusion. This aggregation scenario is referred to as diffusion-limited or reaction-limited aggregation [9-12]. Later models assume an extended aggregation scenario by taking into account the reversible reorganization processes, associated with the breaking away and diffusing of particles from one cluster to another [13-15].

In reality, all of the above mechanisms play a part in the coarsening dynamics. However, in different regimes, different mechanisms dominate. When the distance between clusters is large, then the Ostwald ripening mechanism dominates, but the escape of particles from clusters can be an extremely slow process. However, when the density of clusters is sufficiently high, they interact and cluster motion can dominate. Note too that the Ostwald ripening mechanism depends on the solubility of the clustering particles in the background (dispersing) phase, implying that for strongly phase-separating systems, Ostwald coarsening can be very slow. The cluster motion process also depends strongly on the properties of the dispersing phase and also, in the case of systems of particles moving over a surface, on the nature of the interactions with the surface and the degree of surface roughness, since clusters may become pinned on a rough surface. In this paper, we develop a simple model to study the interplay of the different coarsening processes and the influence of the background substrate.

The model we consider here consists of a system of onedimensional (1D) Brownian particles (i.e., rods) confined in a periodic external potential $U(x)$. Such a situation occurs when particles are confined within a narrow pore, groove, or channel. The amplitude of the modulations in $U(x)$ has a significant influence on the rate at which individual particles 
and also clusters can move through the system. The particles are assumed to have a hard core of diameter $\sigma$ and also an attractive interaction. This system also serves as a minimal model of molecular transport through micro- and nanopores featuring the so-called single file diffusion [16]. We focus, in particular, on the case of two clusters in the system, and we study in detail the dynamics of how these clusters join together. We also focus on small clusters, where it is easier to understand the processes that occur during coalescence. However, we relate our results to larger clusters and we believe our results are quite general.

We find that the Ostwald and translation aggregation mechanisms discussed above are both exhibited by our model system, depending on the values of the system parameters, and we also find that as these are varied, there can be a crossover from one mode dominating to the other. The interplay of these two modes was studied previously for shallow liquid droplets on homogeneous and heterogeneous surfaces [17-21]. There, the analog of the Ostwald process is referred to as the mass transfer mode of the coarsening dynamics of dewetting liquid films [17] or the mass exchange and collapse mode [20]. This refers to when one of two neighboring liquid droplets shrinks and the other one grows, leading eventually to a single large droplet. Similarly, there is a translation mode [17] called the spatial motion and collisions mode in [20], whereby neighboring droplets move together and join. We see here that there are strong parallels between the aggregation dynamics of colloidal (Brownian) systems and the coarsening dynamics in binary mixtures or in dewetting liquid films $[8,17,20]$. The qualitative behavior of the present model is very similar to that observed in these systems, and so we believe that the overall qualitative conclusions we draw in this paper are rather more general than one might expect, given the simple 1D model that we study. This is due to the fact that the coarsening modes are closely related to the symmetry modes of the systems [21].

A particular application of our results is the aggregation in colloidal systems, since we assume the particles move by Brownian dynamics. The rise of nanotechnology has seen a surge of interest in aggregation and cluster formation of colloidal particles with nanoclusters hailed as prototypes of novel functionalized materials [22,23]. The formation of clusters of attracting colloids with their subsequent aggregation into a new solidlike phase is closely linked to the phenomena of glass transitions and gelation [24,25]. At a given volume fraction, the uniform density state, which corresponds to a homogeneous suspension of colloids, can become unstable if a certain critical attraction strength is reached. As the system evolves toward a global minimum of the free energy, the initially formed clusters merge to form bigger aggregates. The final state, which is found in the long-time limit, crucially depends on the nature of the interaction potential between the particles. Typically, if the interaction is purely attractive up to the hard-core cutoff, the global minimum of the free energy corresponds to a single infinitely large aggregate. The process by which large colloidal clusters collapse to form a disordered solid is known as gelation $[11,25,26]$.

To describe the dynamics of cluster aggregation, we take a statistical mechanical point of view and study the kinetics of the aggregation process using dynamical density functional theory (DDFT) [27-30], which is a theory for the time evolution of the one-body density distribution $\rho(x, t)$ of the particles in the system. DDFT builds on equilibrium density functional theory [31-33] and therefore in principle guarantees giving the correct equilibrium fluid density profile, even though its description of the dynamics involves approximation [27-30]. An advantage of DDFT over some other approaches, such as the previously used discrete models [9-15], is that DDFT takes as input the microscopic properties of the particle interactions and particle dynamics. The approximation for the equilibrium Helmholtz free-energy functional that we use is based on the exact free-energy functional for a 1D fluid of hard rods [34]. The contribution to the free energy due to the attraction between the particles is then treated using a simple mean-field approximation [32]. This is based on the DDFT for aggregating hard rods transported along narrow channels developed in Refs. [35,36], where the model is used to study the directed transport of particles through channels with periodically corrugated walls.

The paper is structured as follows: In Sec. II, we give a brief overview of the DDFT for attractive hard rods. In Sec. III, we discuss the bulk system phase behavior, in particular recalling the aspects of the linear stability analysis of the homogeneous phase of the model that are relevant to the present study. In Sec. IV, we discuss how to obtain the eigenfunctions and eigenvalues corresponding to the two different coarsening modes discussed above, and we illustrate this with some typical results. We show that cluster coarsening can be halted by applying a sufficiently strong pinning potential $U(x)$. The critical strength of the potential, which is necessary to stop clustering, greatly depends on the diameter of the particle $\sigma$ and in fact diverges in the limit $\sigma \rightarrow 0$. We show that for small and intermediate amplitudes of the external potential, the aggregation process is dominated by the Ostwald mode if the attraction between particles is rather weak. However, as the attraction strength is increased, the translation mode becomes dominant, whereas the Ostwald mode becomes inactive. In Sec. V, we discuss further how a crossover from one coarsening mode to the other can occur. Finally, in Sec. VI we make a few concluding remarks.

\section{MODEL SYSTEM AND DDFT}

Following [35,36], we consider a system of $N$ hard particles of diameter $\sigma$, moving in a channel of length $S$. We impose periodic boundary conditions, i.e., the channel may be considered to be circular. The channel is assumed to be sufficiently narrow that the particles are confined to a line and so may be treated as a 1D system of hard rods. The particles interact with the corrugated channel walls through a periodic potential $U(x)$, which is of period $L=S / n$, where $n$ is an integer. In addition to the hard-core repulsion between the particles, there is an attractive interaction between the particles that is described by the potential $w(x)=-\alpha \exp (-\lambda x)$, with the typical interaction range $1 / \lambda$ and strength $\alpha>0$. We assume that the dynamics of the particles are governed by an overdamped stochastic equation of motion, and so we may use DDFT [27-30] to approximate the Fokker-Planck equation for the time evolution of the one-body density distribution $\rho(x, t)$ 
as follows:

$$
\frac{1}{\Gamma} \frac{\partial \rho(x, t)}{\partial t}=\frac{\partial}{\partial x}\left[\rho(x, t) \frac{\partial}{\partial x} \frac{\delta F[\rho(x, t)]}{\delta \rho(x, t)}\right],
$$

where $\Gamma$ is the mobility of a single particle, and the Helmholtz free-energy functional $F[\rho]$ is given by

$$
\begin{aligned}
F[\rho(x, t)]= & k_{B} T \int_{-\frac{S}{2}}^{\frac{S}{2}} d x \rho(x, t)[\ln \Lambda \rho(x, t)-1] \\
& +\int_{-\frac{S}{2}}^{\frac{S}{2}} d x U(x, t) \rho(x, t)+F_{\mathrm{hc}}[\rho]+F_{\mathrm{at}}[\rho] .
\end{aligned}
$$

In Eq. (2) the first term is the ideal-gas contribution to the free energy, where $\Lambda$ is the thermal de Broglie wavelength, $k_{B}$ is Boltzmann's constant, and $T$ is the temperature. The term $F_{\text {at }}$ is the excess free-energy contribution due to the attraction between particles, for which we use the following mean-field approximation [32]:

$$
F_{\mathrm{at}}[\rho]=\frac{1}{2} \int_{-\frac{S}{2}}^{\frac{S}{2}} d x \int_{x-\frac{S}{2}}^{x+\frac{S}{2}} d x^{\prime} w\left(\left|x-x^{\prime}\right|\right) \rho(x) \rho\left(x^{\prime}\right) .
$$

The term $F_{\text {hc }}[\rho]$ in Eq. (2) is the free-energy contribution from the hard-core interaction between the particles. We use the Percus exact functional [34] for 1D hard rods of length $\sigma$ :

$F_{\mathrm{hc}}[\rho]=\frac{1}{2} \int_{-\frac{S}{2}}^{\frac{S}{2}} d x \phi[\rho(x)]\left\{\rho\left(x+\frac{\sigma}{2}\right)+\rho\left(x-\frac{\sigma}{2}\right)\right\}$,

where $\quad \phi[\rho(x, t)]=-k_{B} T \ln [1-\eta(x, t)] \quad$ and $\quad \eta(x, t)=$ $\int_{x-\sigma / 2}^{x+\sigma / 2} d x^{\prime} \rho\left(x^{\prime}, t\right)$.

Any solution of Eq. (1) is subject to periodic boundary conditions in the interval $[-S / 2, S / 2]$ and the normalization condition $\int_{-S / 2}^{S / 2} \rho(x) d x=N$.

To nondimensionalize Eq. (1), we rescale $x$ with the characteristic length of the attraction potential $1 / \lambda$, time $t$ with $1 / \lambda^{2} \Gamma k_{B} T$, which represents the characteristic time for a particle to diffuse a distance $1 / \lambda$, and all energy units are scaled with $k_{B} T$. For simplicity, we use the same notations for the rescaled units, implying that nondimensional Eqs. (1)-(4) are obtained by setting $\Gamma=1, \lambda=1$, and $k_{B} T=1$.

We assume for simplicity that the periodic external potential has the following form:

$$
U(x)=-\frac{\chi}{2 \pi} \cos \left(\frac{2 \pi x}{L}\right) .
$$

The amplitude parameter $\chi$ governs the height of the potential barrier between neighboring minima in the potential, and $L$ is the period of the corrugation potential exerted onto the particles by the channel walls. Our simple 1D model may also be used to understand the collective dynamics or particles diffusing over a rough surface. In this context, $\chi$ may be thought of as a parameter that characterizes the roughness of the surface. We also study smooth channels, which correspond to the limit $\chi \rightarrow 0$. In this case, when $U(x)=0$, if the particles condense to form a cluster or several clusters and so are nonuniformly distributed with density profile $\rho(x)$. In this situation the free energy of the system does not change if there is an arbitrary translation in the system, i.e., when $\rho(x) \rightarrow \rho(x+l)$, where $l$ is the arbitrary shift distance. In the numerical treatment of this case, some care has to be taken because of the translation symmetry (see the next section).

\section{PHASE BEHAVIOR AND STABILITY OF THE UNIFORM DENSITY STATE}

In Ref. [36], the equilibrium phase behavior of the model system defined in Eqs. (1)-(4) is studied. As the attraction strength $\alpha$ is increased (or, equivalently, as the temperature $T$ is decreased), the uniform density state $\rho(x)=\rho_{0}$ that exists for $\chi=0$ becomes unstable for some range of densities $\rho_{0}$, and phase separation occurs. While there is no true phase transition in the system as predicted by the present mean-field model because the system is one-dimensional (i.e., fluctuations round the predicted transition), nonetheless a comparison with Brownian dynamics computer simulations reveals that the DDFT (1)-(4) does describe well the aggregation of particles into clusters [36]. A cluster state may form spontaneously if the uniform state is linearly unstable, or there may be a free-energy barrier that must be overcome to form clusters, i.e., clusters must be nucleated [3,36]. A linear stability analysis $[29,36]$ shows that the trivial steady-state solution $\rho(x)=\rho_{0}$ of Eq. (1) can become linearly unstable with respect to two distinct instability modes: the spinodal mode and the freezing mode, depending on the value of $\rho_{0}$. Expanding on the right-hand side of Eq. (1) in powers of the density, one obtains an equation of the form

$$
\partial_{t} \rho=\hat{L} \rho+O\left(\rho^{2}\right)
$$

where $\hat{L}(x)$ is a linear operator. In the linear stability analysis, the density is assumed to be of the form $\rho(x, t)=\rho_{0}+$ $\epsilon \exp [\beta(k) t+i k x]$, where the amplitude $\epsilon$ is assumed to be small. Substituting this into Eq. (6) and then linearizing in $\epsilon$, one obtains the dimensionless dispersion relation [36]

$$
\beta(k)=-k^{2}-\frac{2 \rho_{0} k \sin (k \sigma)}{1-\rho_{0} \sigma}-\frac{4 \rho_{0}^{2} \sin ^{2}(k \sigma / 2)}{\left(1-\rho_{0} \sigma\right)^{2}}+\frac{2 \alpha \rho_{0} k^{2}}{k^{2}+1} .
$$

In the case of an infinitely extended system $(S \rightarrow \infty)$, the spinodal instability sets in at zero wave number $k=0$. In contrast, the freezing instability sets in with a finite critical wave number $k=k_{c} \neq 0$. The linear stability analysis allows for a determination of the regions of the phase diagram where phase separation occurs and identifies the length scales of the structures that are formed in the initial stages after a uniform state is quenched into a linearly unstable parameter region [29,36]. However, here our focus is not on the linear short-time dynamics but on the long-time dynamics when clusters are already present, whether formed by nucleation or spontaneously after a quench. These clusters coarsen over time, via one or both of the two mechanisms described in the Introduction: either the Ostwald ripening mechanism, or via the motion and coalescence of clusters.

To calculate solution branches of steady cluster density profiles and their bifurcations numerically, we employ the method presented in Ref. [36]. Namely, we rewrite our governing integrodifferential equations as a finite-dimensional 
dynamical system, i.e., as a finite set of ordinary differential equations in time. To do so, Eq. (1) is Fourier-transformed truncating at a certain number of modes $M_{f}$, resulting for our gradient dynamics Eq. (1) in $M_{f}$ real equations for the Fourier coefficients that form the dynamical system. Throughout the paper, we set $M_{f}=400$. Its solution and bifurcation behavior are systematically analyzed combining fast Fourier transforms with pseudo-arclength path continuation [37-39] as bundled in the package AUTO07P [40].

The continuation may either be started at a steady state obtained via a direct time simulation or from analytically known small-amplitude harmonic solutions that well approximate nonuniform states close to the primary bifurcations of the uniform state [36]. However, to numerically obtain $S$-periodic steady-state solutions of Eq. (1) in a smooth channel, i.e., the limit $\chi \rightarrow 0$, one has to overcome a problem that is related to the translational invariance of solutions in this case. In practice, we use the homotopy method to obtain a weakly modulated steady state with a period equal to the system size, i.e., we set $L=S$. We start the continuation with a constant density $\rho(x)=\rho_{0}$, which is a steady-state solution when $\alpha=\sigma=0$ (so that $F_{\mathrm{at}}=F_{\mathrm{hc}}=0$ ) and $U(x)=0$. We then introduce an additional homotopy parameter, which multiplies the last three terms in Eq. (2). In the first continuation run, the homotopy parameter is increased from 0 to 1 , keeping $\chi, \alpha$, and $\sigma$ at generic values in the linearly stable parameter region. This results in a weakly modulated steady state. In the second run, this steady state is continued as $\alpha$ is increased beyond the point where condensation sets in and large-amplitude cluster solutions emerge. In the next run, this large-amplitude solution is continued when the heterogeneity amplitude $\chi$ is decreased until it becomes vanishingly small (typically $\chi=10^{-5}$ well approximates a smooth channel). Thus, the potential $U(x)$ of nearly vanishing amplitude serves in this situation merely as a numerical trick to break the degeneracy related to translational invariance and to fix the steady-state solutions in space.

\section{COARSENING OF CLUSTERS}

In this section, we focus on the dynamics of clusters that have been created either by means of nucleation from a metastable uniform state, or by linear instability (spinodal or freezing). In particular, we consider the coalescence of two clusters into one bigger aggregate. Note that clusters can also be created in the absence of the attraction between the particles, by the potential $U(x)$. In this case, the particles form clusters due to accumulation around the local minima of $U(x)$.

\section{A. Symmetry and coarsening modes}

Any steady-state solution of Eq. (1) in a smooth channel, i.e., with $U(x)=0$, has two types of symmetry: (i) a translation symmetry and (ii) a volume change symmetry. The existence of these two symmetries for individual clusters gives rise to the two different coarsening mechanisms discussed above if they are combined for several clusters. This is sketched for droplets on a solid substrate in Ref. [21].

For the translation symmetry, one sees that if $\rho(x)$ is a single-cluster steady-state solution of Eq. (1), then $\rho(x+\xi)$ with an arbitrary shift $\xi$ is also a solution. For small $\xi$, one can write $\rho(x+\xi) \approx \rho(x)+\xi \partial_{x} \rho(x)$, which implies that $h(x)=\partial_{x} \rho(x)$ is the eigenfunction of the DDFT operator $\hat{L}(x)$ linearized around the steady state $\rho(x)$, with zero eigenvalue $\gamma=0$, i.e., $\hat{L}(x) \partial_{x} \rho(x)=0$. In what follows, we refer to $h(x)=\partial_{x} \rho(x)$ as the translation symmetry mode eigenfunction, which is sometimes called the Goldstone mode of this continuous symmetry. Combining translation modes with opposite signs for two adjacent droplets gives the translation mode of coarsening.

The volume change symmetry is associated with the normalization (particle conservation) condition of the solution of Eq. (1). Given a density profile $\rho(x)$, which is a singlecluster steady-state solution of Eq. (1), with

$$
\frac{1}{S} \int_{-S / 2}^{S / 2} \rho(x) d x=\rho_{0},
$$

we now consider the density profile $\tilde{\rho}(x)=\rho(x)+\delta \rho(x)$, with $(1 / S) \int_{-S / 2}^{S / 2} \tilde{\rho}(x) d x=\tilde{\rho}_{0}=\rho_{0}+\delta \rho_{0}$, which is also a steady-state solution of Eq. (1) for some small $\delta \rho_{0}$. Since $\tilde{\rho}(x)=\rho(x)+\delta \rho(x)$, we deduce that $h(x)=\delta \rho(x)$ is another eigenfunction of the operator $\hat{L}(x)$ with zero eigenvalue $\gamma=0$, i.e., $\hat{L}(x) \delta \rho(x)=0$. Consequently, the volume symmetry mode $\delta \rho(x)$ corresponds to the derivative with respect to the density $\rho_{0}$, i.e., $\delta \rho(x)=\delta \rho_{0} \partial \rho(x) / \partial \rho_{0}$, where we define

$$
\frac{\partial \rho(x)}{\partial \rho_{0}} \equiv \lim _{\delta \rho_{0} \rightarrow 0} \frac{\rho\left(x ; \rho_{0}+\delta \rho_{0}\right)-\rho\left(x ; \rho_{0}\right)}{\delta \rho_{0}} .
$$

Combining volume modes with opposite signs for two adjacent droplets gives the mass transfer mode of coarsening, which we refer to as the Ostwald ripening mode. Note that one may alternatively calculate the Ostwald mode eigenfunction by considering the change $\delta \rho(x)$ from an infinitesimally small change in the value of the chemical potential, $\mu_{0} \rightarrow \mu_{0}+\delta \mu_{0}$.

Thus, any steady-state solution $\rho(x)$ has the two neutrally stable symmetry modes. If these respective modes are combined for pairs or groups of clusters, one obtains the various coarsening mode eigenfunctions that for homogeneous systems normally have small positive eigenvalues resulting from the overlap of the tails of the symmetry modes of the individual clusters. If one now breaks the translation symmetry by considering a heterogeneous system with $U(x) \neq 0$, the stability and characteristics of translation and Ostwald coarsening modes closely related to the two introduced eigenfunctions still define the dynamics of coarsening.

To illustrate this discussion, one may examine the simple situation in which an ideal gas of noninteracting particles (i.e., $\sigma=0$ and $\alpha=0)$ is confined within a parabolic potential $U(x)=a x^{2}$, where $a$ determines the strength of the confinement. This is arguably the simplest example of a cluster, where the particles remain in the cluster due to the confinement of the external potential. The equilibrium density profile is obtained from minimizing the grand potential [32],

$$
\Omega[\rho]=F[\rho]-\mu \int_{-S / 2}^{S / 2} \rho(x) d x,
$$

where the Helmholtz free energy $F$ results from Eq. (2), and $\mu$ is the chemical potential, which is the Lagrange multiplier enforcing the constraint (8). When $S$ is large, we obtain the density profile $\rho(x)=\rho_{0} \exp \left(-a x^{2}\right)$, where 
$\rho_{0}=\Lambda^{-1} \exp (\mu)$. Thus, the translation mode eigenfunction is $h(x)=\partial_{x} \rho(x)=-2 x a \rho_{0} \exp \left(-a x^{2}\right)$. To determine the volume mode eigenfunction, we note that $\rho\left(x ; \rho_{0}+\delta \rho_{0}\right)=$ $\left(\rho_{0}+\delta \rho_{0}\right) \exp \left(-a x^{2}\right)$, and so using Eq. (9) we find that the volume mode eigenfunction is $h(x)=\delta \rho(x) / \delta \rho_{0}=\exp \left(-a x^{2}\right)$. Note that the translation and volume mode eigenfunctions are orthogonal. We now consider two such confining potentials generating two clusters a distance $l$ apart. In this situation, $U(x)=a(x+l / 2)^{2}$ for $x<0$ and $U(x)=a(x-l / 2)^{2}$ for $x>0$. The resulting density profile exhibits two "clusters" centered at $\pm l$ and the density profile

$$
\rho(x)=\left\{\begin{array}{lll}
\rho_{0} \exp \left[-a(x+l / 2)^{2}\right] & \text { for } \quad x<0, \\
\rho_{0} \exp \left[-a(x-l / 2)^{2}\right] & \text { for } \quad x>0 .
\end{array}\right.
$$

Suppose now that there are slightly more particles $\left(\delta \rho_{0}\right)$ in the left-hand cluster than in the right-hand cluster. In this situation, the Ostwald mode eigenfunction is

$$
h(x) \approx \begin{cases}\exp \left[-a(x+l / 2)^{2}\right] & \text { for } x<0, \\ -\exp \left[-a(x-l / 2)^{2}\right] & \text { for } x>0 .\end{cases}
$$

This eigenfunction indicates that there will be a diffusion of particles from one side to the other. One must calculate the eigenvalue to determine the direction of this flux, although in this simple situation one knows already that the symmetric state is stable, i.e., the eigenvalue must be negative and the flux is from left to right. One may also examine the translation mode eigenfunction, although in this simple case this mode is not relevant. However, when we introduce the attraction between the particles, this mode can become relevant and the two clusters can seek to move toward one another.

We now consider a small system of strongly interacting particles. We display in Fig. 1(a) a single four-particle cluster, i.e., a period- $L$ steady-state density profile obtained for $\sigma=0.75, \alpha=15, \rho_{0}=0.8$, and $S=L=5$. Due to the very strong attraction, the four particles are squeezed
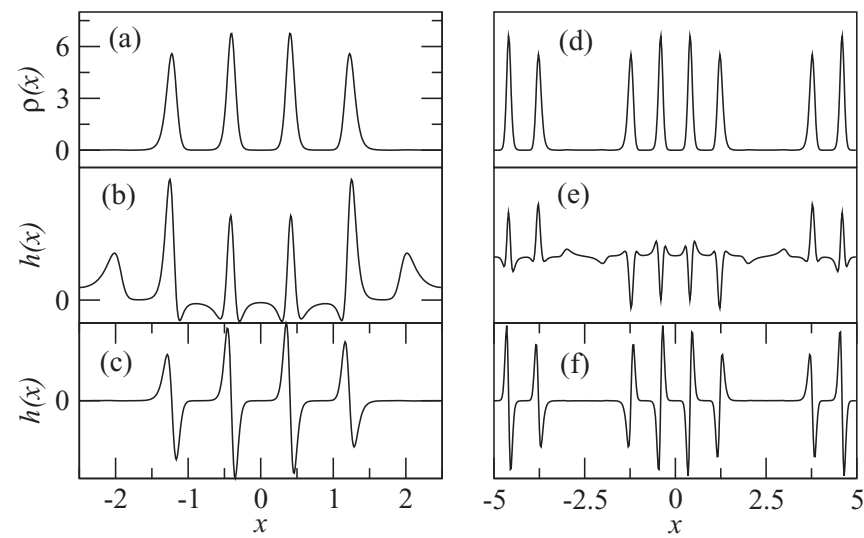

FIG. 1. (a) Density profile for a single cluster that is a period$L$ steady-state solution in the smooth channel $[U(x)=0]$ for $\rho_{0}=$ $0.8, \alpha=15, \sigma=0.75$, and domain size $S=L=5$. (b) The volume symmetry mode and (c) translation symmetry mode eigenfunctions $h(x)$ for a single cluster with eigenvalues $\gamma=0$. (d) Density profile for a two-cluster state, i.e., period- $L$ steady-state solution in a domain of length $S=2 L=10$, for parameters as in (a). (e) Ostwald ripening coarsening mode with $\gamma=-0.8$ (stable). (f) Translation coarsening mode with $\gamma=3.5$ (unstable). into the compact cluster with four distinct density peaks. Figures 1(b) and 1(c) show the volume symmetry mode and the translation symmetry mode eigenfunctions of the steady state, respectively.

If $L$ is the period of a (neutrally) stable state $\rho(x)$, then the periodically continued $\rho(x)$ is also a steady-state periodic solution in a system with the larger size $S=n L$, with an arbitrary integer $n$. In such an extended system, the solution $\rho(x)$ is still (neutrally) stable with respect to perturbations with period $L$, but it may be unstable with respect to perturbations with period $n L / m(m=1, \ldots, n)$ that break the internal discrete symmetry of $\rho(x)$ under translations by $L$, i.e., to coarsening modes.

Here we are particularly interested in the case of $n=2$, i.e., when $S=2 L$. We refer to the period- $L$ steady state $\rho(x)$ as a cluster of length $L$. Then, the case $n=2$ corresponds to two identical clusters of period- $L$ in a system of total size $S=2 L$. Figure 1(d) shows the density profile for such a two-cluster state, i.e., two period- $L(L=5)$ clusters of four particles each in a system of size $S=2 L=10$. In the following sections, we describe the coalescence dynamics of the two period- $L$ clusters that are stable with respect to perturbations with wavelength $L$, but may be unstable with respect to perturbations with wavelength $2 L$. These (stable or unstable) period- $2 L$ perturbations are the two coarsening modes discussed above. The mode associated with the Ostwald ripening coarsening process can be approximated by combining the volume modes of two individual clusters taken with opposite signs, as shown in Fig. 1(e). See also Eq. (12). The second mode is the translation coarsening mode, which is obtained by combining the translation modes of the individual clusters taken with opposite signs. This is shown in Fig. 1(f). For the case in Fig. 1, the eigenvalue for the Ostwald coarsening mode, $\gamma=-0.8$, is negative and so the system is stable against this mode. However, for the translation mode, $\gamma=3.5$, and so the system is unstable against this mode. Because this mode is unstable, the two clusters move toward each other, preserving their shape until they collide to form a bigger cluster.

\section{B. Purely attracting particles}

First, we consider the simplest case of particles with $\sigma=0$, i.e., with no hard-core interaction. We set $\rho_{0}=1 / L$ and $S=$ $2 L$, corresponding to just one particle per period- $L$, i.e., we expect "clusters" with either one or two particles. Figure 2(a) shows the $L_{2}$ norm of the steady states of Eq. (1) as a function of $\alpha$, obtained for $L=5$. We see that the period- $L$ solution, which for $\alpha \lesssim 6$ corresponds to a weakly modulated density distribution [see the inset Fig. 2(a)], undergoes two primary pitchfork bifurcations: one at $\alpha \approx 3.3$ and the other at $\alpha \approx 3.8$, where two new branches of steady-state period- $2 L$ solutions emerge, as shown by the red dot-dashed line and by the black solid line in Fig. 2(a). The first one, bifurcating at $\mathrm{BP}_{1}$, consists of stable period- $2 L$ solutions that represent strongly localized two-particle clusters centered at the minimum of $U(x)$. The branch that emerges at $\mathrm{BP}_{2}$ consists of unstable localized twoparticle clusters (unstable period- $2 L$ solutions) centered at the maximum of $U(x)$ [see the inset of Fig. 2(a)].

At each pitchfork bifurcation, the period- $L$ becomes unstable with respect to one of the coarsening modes, i.e., for 

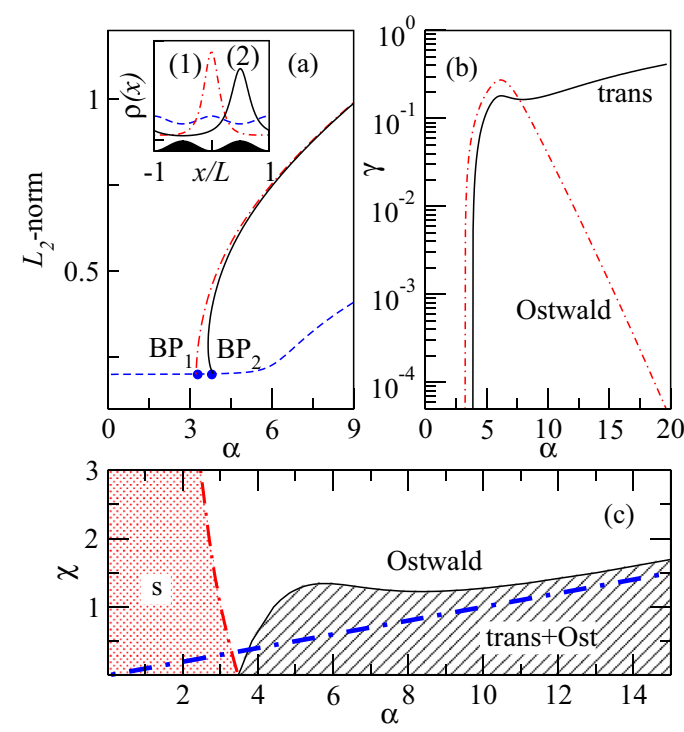

FIG. 2. (Color online) (a) The $L_{2}$-norm of the steady-state solutions of Eq. (1) for $\chi=0.5, \rho_{0}=1 / L$, and $S=2 L=10$. The dashed line corresponds to period- $L(L=5)$ solutions; the dotdashed line and the solid line correspond to period- $2 L$ solutions. Two branches of period- $2 L$ solutions emerge at the pitchfork bifurcations $\mathrm{BP}_{1}$ and $\mathrm{BP}_{2}$. The inset shows the channel potential (shaded) and three density profiles: the period- $L$ solution (dashed line) obtained for $\alpha=3.5$, the period- $2 L$ solution on the first branch at $\alpha=3.4$ (dot-dashed line), and the period- $2 L$ solution on the second branch (solid line) at $\alpha=3.8$. (b) The two leading eigenvalues $\gamma$ of the period- $L$ solutions: the Ostwald mode and the translation mode (trans). All other parameters are as in (a). (c) Stability diagram of the two coarsening modes in the $(\chi, \alpha)$ plane. Period- $1 L$ solutions are stable in the shaded area marked by $(s)$. The translation mode is stable above the solid line. The Ostwald mode (Ost) is always unstable outside the area marked by $(s)$. The thick dot-dashed line corresponds to the estimate for the stability threshold of the translation mode given by Eq. (13).

$\alpha \gtrsim 3.8$, the period- $L$ solution is unstable with respect to the two coarsening modes described above. At the first branching point $\mathrm{BP}_{1}$, the system becomes unstable to the Ostwald ripening mode. Indeed, the centers of the two period- $L$ clusters induced by the potential $U(x)$ are located in the minima of $U(x)$. Therefore, the position of the clusters does not change as one switches to the first branch. In contrast, when moving from the period- $L$ solution onto the second branch (above its saddle-node bifurcation), the position of clusters changes from the minimum to the maximum of $U(x)$, clearly indicating that the second branching point $\mathrm{BP}_{2}$ corresponds to the onset of the translation mode of coarsening. Note that at large $\alpha$, the norms of the two branches of solutions practically coincide.

The two leading eigenvalues $\gamma$ of the steady period- $L$ solutions are shown in Fig. 2(b). They become positive at the respective pitchfork bifurcation. For $\alpha$ not too far above these thresholds, the Ostwald mode (dot-dashed line) dominates over the translation mode (solid line). However, as $\alpha$ is further increased, the eigenvalue of the Ostwald mode rapidly decreases, whereas the eigenvalue of the translation mode increases almost linearly with $\alpha$. This shows that for strongly attractive particles, the translation mode dominates the cluster coalescence process.

The stability diagram in Fig. 2(c) shows the locus of the two branching points $\mathrm{BP}_{1}$ and $\mathrm{BP}_{2}$ in the $(\alpha, \chi)$ plane. The red dot-dashed line marks the first bifurcation of the period- $L$ state, associated with the onset of the Ostwald mode. The black solid line corresponds to the onset of the translation mode. The lefthand shaded region corresponds to the linearly stable period- $L$ state. Note that for any fixed $\alpha$, one can stabilize the translation mode by an external potential $U(x)$ with a sufficiently large amplitude $\chi$. Remarkably, however, the Ostwald mode cannot be stabilized for any choice of $\chi$, implying that two period$L$ clusters will always eventually merge as a result of the diffusion process that is the basis of the Ostwald coarsening mechanism. We emphasize, however, that the characteristic time scale $\tau \sim 1 / \gamma$ associated with the Ostwald mode diverges exponentially for large $\alpha$, as can be clearly seen from Fig. 2(b), where $\gamma$ is plotted on a logarithmic scale. This means that for strongly attractive particles, the transfer of material from one cluster to the other via the Ostwald mode is a very slow process, as one would expect for particles diffusing over a high potential barrier.

One can give a rather simple qualitative estimate for the stability threshold of the translation mode [dashed line in Fig. 2(c)]. This can be done by relating the peaks in the density profile to individual particles. Thus, we associate the period- $L$ cluster with $N=\rho_{0} L$ particles located at the minima of $U(x)$, as shown by the dashed lines in Fig. 3. Since the attraction strength between particles decreases exponentially with distance, we only need to take into account the interactions between nearest-neighbor particles (clusters). When two neighboring clusters move toward each other by a distance $\delta$, then the total interaction energy $E(\delta)$ per cluster can be written as $E(\delta)=\rho_{0} L\left\{-\alpha e^{-(L-2 \delta)}+0.5\left[U\left(x_{\min }+\right.\right.\right.$ $\left.\left.\delta)+U\left(x_{\min }-\delta\right)\right]\right\}$. By setting $E^{\prime \prime}(0)=0$, we find that the equilibrium configuration $\delta=0$ is stable with respect to the translation mode when

$$
\pi \chi \geqslant 2 \alpha L^{2} e^{-L}
$$

The critical amplitude $\chi$ from Eq. (13) is displayed as the thick dot-dashed line in Fig. 2(c), which slightly underestimates the true stability threshold (solid line) because Eq. (13) is obtained via purely energetic arguments and does not take into account entropic contributions.

The physical reason why the Ostwald coarsening process for particles with no hard core $(\sigma=0)$ cannot be stabilized by

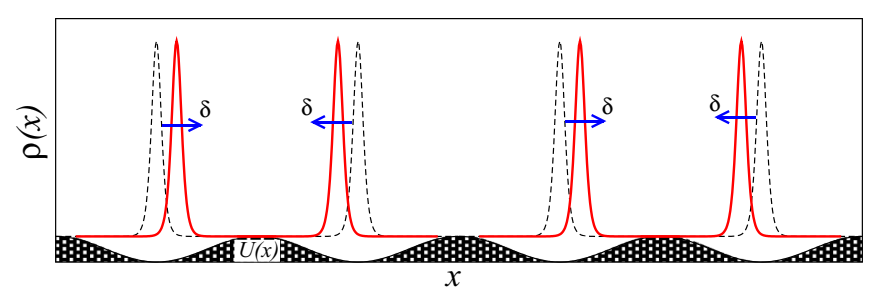

FIG. 3. (Color online) Schematic representation of the translation coarsening mode. The initial clusters (dashed lines) move by a distance $\delta$ toward one of their neighbors. The external potential $U(x)$ is indicated by the shaded profile at the bottom. 
increasing the amplitude of the pinning potential $U(x)$ to some threshold value can be explained as follows: Particles with no hard-core repulsion can always be squeezed into a cluster of arbitrarily small size. So, initially, strong periodic potentials $U(x)$ split the particles into dense clusters, each located at a local minimum of $U(x)$. However, even in the limit $\chi \rightarrow \infty$, the question of the stability of the dense clusters that are located in the minima of $U(x)$ becomes the standard escape problem of a single particle from a potential well. The effective energy barrier that a given particle must overcome in order to escape a cluster is given by the combination of the pinning potential and the energy associated with the attraction to the cluster. It is well known that the escape rate always remains nonzero, no matter how high the potential barrier is. This ensures that the temperature-driven Ostwald mode diffusive process remains active even for very strongly pinning potentials $U(x)$. Intuitively, however, one may expect this mechanism to break down in the case in which the particles do have a hard core $(\sigma>0)$, as these can no longer be squeezed into a cluster below the size of $\sigma N$.

\section{Stabilization of clusters by strongly corrugated potentials}

Under similar conditions, hard rods with nonzero length $\sigma \neq 0$ behave qualitatively different from the pointlike purely attracting particles considered in Sec. IV B. Even an arbitrarily small $\sigma$ has a dramatic effect on the stability diagram displayed in Fig. 2(c). For any $\sigma \neq 0$, the Ostwald mode can be stabilized by a sufficiently strong corrugation potential $U(x)$. Note that, in the large $\chi$ regime, the translation mode is also stable as shown for $\sigma=0$ in Sec. IV B. As a result, for any $\sigma \neq 0$ a strong pinning potential can stabilize clusters with a size sufficiently small to fit within a single potential well of the channel potential.

To see why large amplitudes $\chi$ can lead to the stabilization of clusters, we consider the strong attraction limit $\alpha \rightarrow \infty$, where the particle picture can be used for qualitative arguments. If there are only $N=2$ particles within the system, one finds four different steady-state solutions of Eq. (1), as shown schematically in Fig. 4. The two rods are indicated by rectangles and the corresponding density distributions are indicated as well. The steady states of these two hard rods consist of the individual particles either being at the minima [Fig. 4(a)] or maxima [Fig. 4(b)] of the channel potential $U(x)$, or they can form a compact two-particle cluster centered at

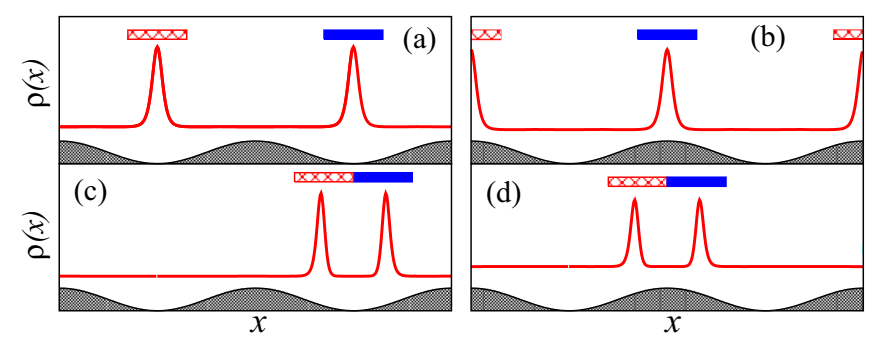

FIG. 4. (Color online) Schematic diagram of the steady-state solutions (solid lines) of Eq. (1) with $N=2$ strongly attracting hard rods with $\rho_{0}=1 / L$ and $S=2 L$. The shaded area represents $U(x)$. The rod length is $\sigma=1.5$.
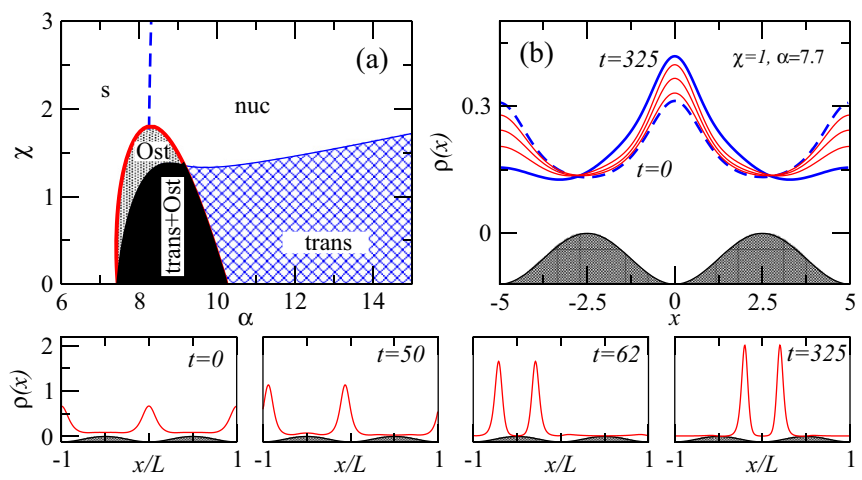

FIG. 5. (Color online) (a) Stability diagram of the two coarsening modes for $\sigma=1.75$ with the remaining parameters as in Fig. 2(c). (b) The coalescence time evolution of two period- $L$ clusters into a single period- $2 L$ cluster mediated by the Ostwald mode, for $\alpha=7.7$ and $\chi=1$. Bottom row: an example of the aggregation dynamics via the translation mode, in this case for $\alpha=10$ and $\chi=1$.

the minimum [Fig. 4(c)] or maximum [Fig. 4(d)] of $U(x)$. Clearly, the steady states (b) and (d) are unstable, as any small deviation from the equilibrium will result in a translation to an energetically lower state [either (a) or (c)].

The energies of the states (a) and (c) are given by $E_{a}=2 U\left(x_{\min }\right)-\alpha e^{-L}$ and $E_{c}=U\left(x_{\min }+\sigma / 2\right)+$ $U\left(x_{\min }-\sigma / 2\right)-\alpha e^{-\sigma}$, respectively. Consequently, taking into account that $U(x)=-(\chi / 2 \pi) \cos 2 \pi x / L$, the state (a) corresponds to a global minimum of the energy for $\chi>\pi \alpha\left(e^{-\sigma}-e^{-L}\right) /(1-\cos \pi \sigma / L)$. Therefore, when $\chi$ exceeds this threshold value, the clusters with $N=1$ particles, associated with the period- $L$ solutions, are linearly stable.

It should be emphasized that the energy-based arguments cannot be used to accurately predict the linear stability threshold. Generally, stabilization occurs at a much lower $\chi$ value, due to the fact that a steady state can be linearly stable even if it has a higher energy than some other steady state. The border of the region in the $(\alpha, \chi)$ plane, where linearly stable period- $2 L$ solutions exist, is given by the dashed line in Fig. 5(a), which presents the modification of the stability diagram in Fig. 2(c) due to a nonzero rod length $\sigma=1.75$.

In the heavy-shaded region of Fig. 5(a) labeled "trans+Ost," both coarsening modes are linearly unstable. The thick red solid line defines the boundary of the region where the Ostwald mode is linearly unstable. In the hash-shaded area, only the translation mode is linearly unstable whereas the Ostwald mode is linearly stable. Outside of the shaded areas, the period- $L$ solutions are linearly stable. However, as the primary bifurcations can be subcritical, the period- $2 L$ clusters can exist outside the shaded area. This occurs to the right of the nearly vertical dashed blue line, which marks the saddle-node bifurcation of the stable period- $2 L$ solutions. In this region, labeled "nuc," the period- $2 L$ clusters must be formed via nucleation. Remarkably, we see from Fig. 5(a) that at nonzero $\chi$, one can switch between the translation mode for coarsening and the Ostwald mode by changing $\alpha$.

Numerically integrating Eq. (1), we obtain the time evolution of the density profile, with the period- $L$ solution as 
the initial condition as displayed in Fig. 5(b). We see that for $\alpha=7.7$ and $\chi=1$, the dynamics of the coalescence of two period- $L$ clusters is indeed via the Ostwald mode. As expected from Fig. 5(a), in this scenario one of the maxima of the density profile grows at the expense of the other. Increasing $\alpha$, we find that the translation mode becomes dominant. In the example displayed along the bottom row in Fig. 5, which is for $\alpha=10$ and $\chi=1$, we observe a typical coalescence dynamics due to the translation mode. The two unstable clusters at the potential minima first both move toward the separating maximum, where they coalesce into the solution on the $\mathrm{BP}_{2}$ branch. Note that this solution is itself unstable (on a larger time scale), as it represents a saddle in function space. However, it acts as an organizing center for the coarsening process as it first attracts the time evolution along its stable direction (related to its stable eigenvalue closest to zero), and then expels it along its only unstable direction (in function space) on a time scale controlled by its unstable eigenvalue. As a consequence, the fused cluster slowly translates into one of the potential minima, becoming the solution on the $\mathrm{BP}_{1}$ branch. In other words, the time scale of the coarsening is controlled by the eigenvalues of the initial two-cluster state and of the unstable solution on the $\mathrm{BP}_{2}$ branch. Overall, the eigenvalue with the smallest absolute value gives a good estimate for the time scale of the coalescence.

\section{Larger clusters}

Up to this point, we have focused on the coalescence of two "clusters" which each only consist of $N=1$ particle (on average). The fair question arises as to whether or not the results summarized in Fig. 5 remain at least qualitatively valid for bigger clusters of $N>1$ particles. The complete answer to this question depends on the choice of the length scale parameters such as the rod length $\sigma$, the period $L$ of the channel potential, and the system size $S$, all expressed in units of $1 / \lambda$. Indeed, by increasing the number of particles in the system, i.e., by increasing the average density $\rho_{0}$, one increases the minimal possible size of the cluster, given by $\sigma N$. Clearly, one can expect to uncover a rather different behavior for $\sigma N \ll L$, where the cluster size is much smaller than the period of $U(x)$ and for $\sigma N \sim L$, with cluster sizes comparable to the period of $U(x)$. Furthermore, different dynamical regimes can be expected for different separation distances between the clusters as compared to their size.

In view of this, it is clear that a complete account of all the possible dynamical regimes of the cluster formation is beyond the scope of the present paper. Here we focus on the simplest case in which the smallest cluster size $\sigma N$ is much smaller than both the period of $U(x)$ and the separation distance between the clusters.

Under such conditions, the energy-based arguments used above for the case of $N=2$, as illustrated in Figs. 3 and 4 , remain qualitatively valid for $N>2$. This means that the stabilization of both coarsening modes by a sufficiently strong potential $U(x)$ can be expected for larger clusters as well. However, the presented energy-based arguments do not explain a stabilization of the Ostwald mode observed even in the case when the channel walls are smooth, i.e., for $\chi=0$ [see Figs. 5(a) and 6]. To understand this effect, we set $\sigma=0.5$ and study the dependence of the coarsening modes on attraction strength $\alpha$ for different numbers of particles in the system as controlled by $\rho_{0}$.

Representative examples are shown in Figs. 6(a) and 6(b), where the eigenvalues of the translation mode and the Ostwald mode are denoted by the solid and dot-dashed lines,
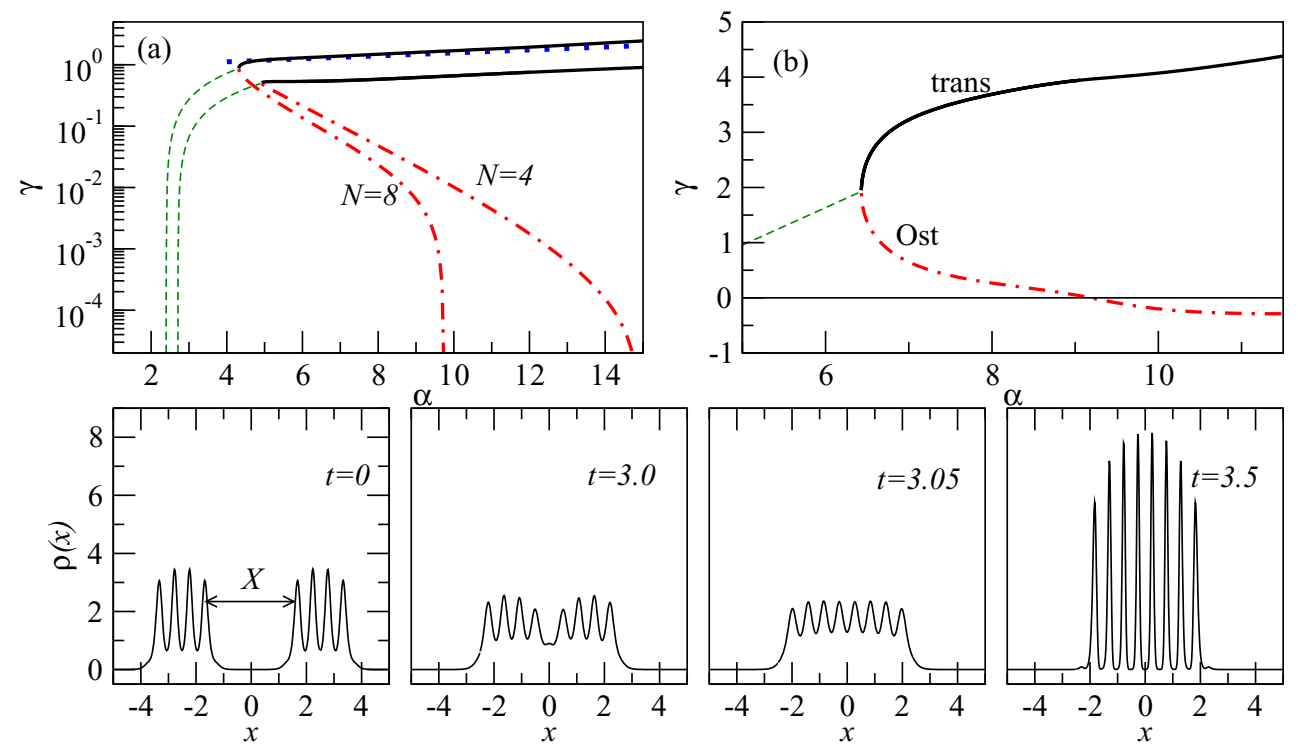

FIG. 6. (Color online) Panels (a) and (b) show the dependence on attraction strength $\alpha$ of the eigenvalues of the translation (thick solid lines) and the Ostwald (dot-dashed lines) coarsening modes of period- $L$ solutions in the case of smooth channels $(\chi=0)$, rodlike particles with $\sigma=0.5$, and domain size $S=2 L=10$. The thin dashed lines correspond to the growth rate $\beta(k)$ of the uniform density state calculated at $k=\pi / 5$. Part (a) gives results for densities $\rho_{0}=0.4$ (i.e., $N=4$ ) and $\rho_{0}=0.8$ (i.e., $N=8$ ), while (b) shows the case $\rho_{0}=1.2$ (i.e., $N=12$ ). The thick dotted line in (a) is a linear fit to the increasing part of the translation mode eigenvalue. The bottom row illustrates the coalescence process for the $\rho_{0}=0.8$ case in (a) at $\alpha=10$, i.e., two $N=4$ clusters coalesce via translation into a single $N=8$ cluster. 


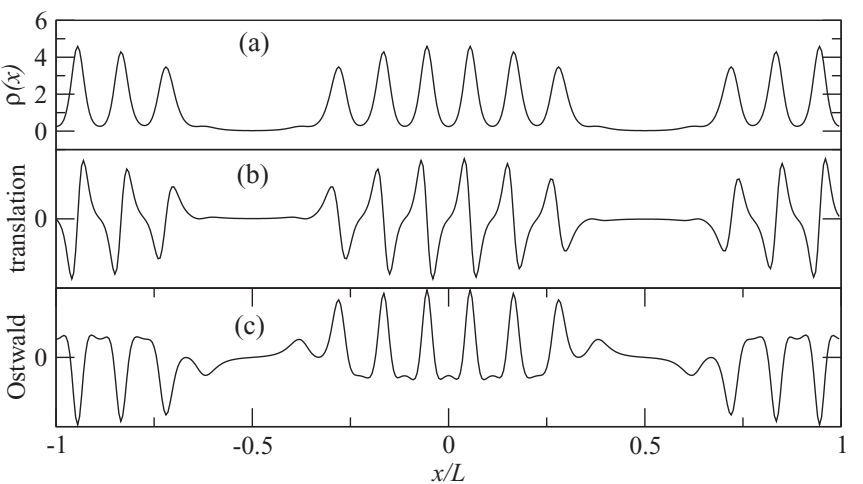

FIG. 7. (a) Steady-state period- $L$ density profile for $S=2 L$ at $\alpha=10$ and other parameters as in Fig. 6(b). (b) The unstable translation coarsening mode with eigenvalue $\gamma=4$. (c) The stable Ostwald coarsening mode with $\gamma=-0.2$.

respectively. Fixing the period $L$ and domain size $S=2 L$, one notes that the critical value of the attraction strength, at which the Ostwald mode becomes stable, depends on the edge-to-edge separation $Y$ between the clusters. Indeed, for $N=4$ (i.e., for $S=10$, an edge-to-edge separation of $Y \approx L-N \sigma=4$ ), the stabilization occurs at around $\alpha \approx 15$, and for $N=8$ (edge-to-edge separation of $Y \approx 3$ ) it occurs at $\alpha \approx 10$. For an even smaller edge-to-edge separation of $Y=2$, the critical attraction strength required for stabilization is $\alpha \approx 9$, as shown in Fig. 6(b). In all cases, the eigenvalue of the translation mode increases at large $\alpha$ monotonically with $\alpha$.

The stabilization of the Ostwald mode in the large- $\alpha$ limit is due to the strong attraction that binds the outer particles strongly to the cluster so that they cannot diffuse away, even under the influence of a neighboring cluster. Thus, for coalescence to occur for large $\alpha$, it must occur via the translation mode. A typical coalescence scenario of two period- $L$ clusters is illustrated for $N=8$ and $\alpha=10$ by the snapshots from a time simulation shown in the bottom row of Fig. 6. At $t=0$, the clusters start to move toward each other due to the dominating unstable translation mode. As the edge-to-edge distance between the clusters decreases, the strength of the attractive interaction increases exponentially $[\sim \alpha \exp (-Y)]$. Initially, the clusters move toward each other rather slowly, but after a certain time $(t=3)$ the increasing attractive forces become rather strong, and the remaining stage of the coalescence is a very quick process with the typical time scale of $10^{-2}$ as compared to $10^{0} \ldots 10^{-1}$ for the initial slow approach. Eventually at $t \approx 3.05$, one larger period- $2 L$ cluster is formed with $N=8$ particles.

For $N=12$, the edge-to-edge separation distance between the period- $L$ clusters is $Y=L-6 \sigma=2<6 \sigma=3$. The steady-state solution as well as the unstable translation and the stable Ostwald coarsening modes are shown for $\alpha=$ 10 in Fig. 7. For small edge-to-edge separations between clusters, i.e., for $Y \ll \sigma N$, the Ostwald coarsening process is suppressed by the close proximity of the clusters.

\section{CROSSOVER BETWEEN COARSENING MODES}

As shown in the previous section, for a fixed center-tocenter distance between the clusters, the translation mode

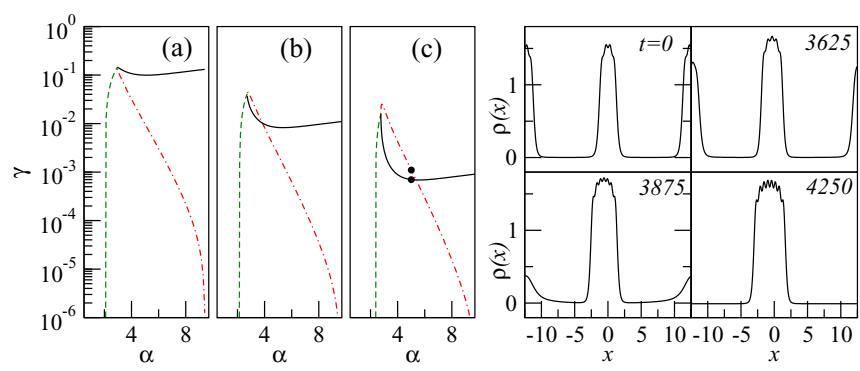

FIG. 8. (Color online) Panels (a)-(c) show for different cluster distances the two eigenvalues of the coarsening modes for a pair of four-particle clusters (period $L=S / 2$ solution) in a channel with smooth walls $(\chi=0)$ as a function of attraction strength $\alpha$. The solid lines are for the translation mode and the dot-dashed lines for the Ostwald mode. The parameters are $\sigma=0.5$ and (a) $S=15$, $\rho_{0}=8 / 15$; (b) $S=20, \rho_{0}=8 / 20$; and (c) $S=25, \rho_{0}=8 / 25$. The filled circles in (c) mark the eigenvalues at $\alpha=5$. The dashed lines are obtained from Eq. (7) for $k=2 \pi / S=\pi / L$. Right panel: time series of density profiles for the aggregation of two clusters induced by the dominant Ostwald mode, calculated for $S=25, \rho_{0}=8 / 25$, and $\alpha=5$.

dominates the aggregation process for large $N$ and $\alpha$. Remarkably, however, the time scale associated with the two coarsening modes changes dramatically, resulting in a partial crossover between the Ostwald and the translation mode, if the separation distance is gradually increased.

To illustrate what we mean by "partial crossover" between the modes, we consider the case with smooth channel walls $\chi=0$ and fix the number of particles in the system to $N=8$, i.e., we look at the coarsening of two clusters of $N=4$ particles each. We then follow the branch of period- $L$ solutions in parameter $\alpha$ for different separation distances that we control via the domain size $S$. This implies that for increasing $S$, we have a decreasing $\rho_{0}=N / S$. The center-to-center distance $X$ between the two clusters is then given by $X=S / 2$.

The two coarsening eigenvalues are shown in Figs. 8(a)8 (c) as a function of $\alpha$ for three different separations $X$. For $X=7.5$ [Fig. 8(a)], the translation mode dominates in the entire $\alpha$ range where the coarsening modes are active $(\alpha \gtrsim 3)$ and there is a strong separation of the time scales $1 / \gamma$ of the two coarsening modes beyond the minimum of the eigenvalue of the translation mode at $\alpha \approx 5$. The Ostwald mode stabilizes at $\alpha \approx 10$. However, at the larger distance $X=10$ [Fig. 8(b)], the Ostwald mode dominates the aggregation process for a relatively weak attraction $3<\alpha<4$, and a crossover between the coarsening modes occurs at $\alpha \approx 4$, with the translation mode dominating for stronger attraction. As the separation distance $X$ is further increased [see, e.g., $X=12.5$ in Fig. 8(c)], the crossover point is shifted toward ever larger values of $\alpha$.

An example of the dynamics of coalescence of two $N=4$ clusters is shown for $S=25(X=12.5)$ and $\alpha=5$ in the right panel of Fig. 8. There, the Ostwald mode $(\gamma=0.0011)$ acts on a shorter time scale $(\sim 1 / \gamma)$ than the translation mode $(\gamma=0.0007)$, as indicated by filled circles in Fig. 8(c). As coarsening progresses, the positions of the clusters remain practically unchanged. Due to the diffusion of particles from one cluster to the other, which characterizes the Ostwald mode, 
one of the clusters shrinks whereas the other one increases in size. Eventually, one large clusters with $N=8$ particles (period-2L solution) is formed.

To decide which mode is dominant under which conditions, one can use the following qualitative argument, which is valid for strongly attracting particles. The time scale associated with the Ostwald mode can be estimated as follows: The binding energy $w_{b}$ of a single rod that joins or leaves the cluster is independent of the number of particles in the cluster, provided that this number is rather large. Therefore, the escape rate $v_{\mathrm{esc}}$ of the particles at the surface of the cluster is given by $v_{\text {esc }} \sim \exp \left(-w_{b}\right)$. Assuming that only one particle can escape from the cluster at a time, we estimate the characteristic time $t_{\mathrm{Ost}}^{\mathrm{esc}}$, which is needed for $N$ particles to escape from the cluster,

$$
t_{\text {Ost }}^{\text {esc }} \sim N \exp \left(w_{b}\right) .
$$

After the particle has escaped, it still needs to diffuse the distance $X$ between the two clusters in order to join the other cluster. The diffusion time is estimated as $t_{\mathrm{Ost}}^{\mathrm{diff}} \sim X^{2}$.

To estimate the time scale associated with the translation mode, we note that two clusters of $N$ particles each can move and collide as a result of the attraction between the clusters. This scenario resembles standard diffusion-limited aggregation [9-12] with an additional migration of clusters due to attraction. Thus, if $X \gg \sigma N$, the attraction force between the clusters is proportional to $N^{2}$, i.e., $f \sim-N^{2} w^{\prime}(X)$, where $w^{\prime}(X)$ is the derivative of the pair potential. Therefore, the force per particle is $f / N \sim-N w^{\prime}(X)$.

The diffusion coefficient $D_{N}$ of a compact cluster of $N$ particles is smaller than the diffusion coefficient of a single particle by a factor of $\sqrt{N}$, i.e., $D_{N}=1 / \sqrt{N}$. Consequently, the time scale of the translation mode is influenced by the time scale due to attraction $t_{\text {trans }}^{\text {at }}$ and the time scale due to cluster diffusion $t_{\text {trans }}^{\text {diff }}$. If they are very different, the smaller one will dominate. In the overdamped limit, we obtain

$$
t_{\text {trans }}^{\text {at }}=\frac{X \exp (X)}{N \alpha}, \quad t_{\text {trans }}^{\mathrm{diff}}=\sqrt{N} X^{2},
$$

where we have used the exponential pair potential $w(X)=$ $-\alpha \exp (-|X|)$.

The time scale estimates in Eqs. (14) and (15) can now be compared to the numerical findings reported in Figs. 6 and 8. It is clear that the dependence on the cluster size is estimated to $t_{\text {Ost }}^{\text {esc }} \sim N \gg t_{\text {trans }}^{\text {diff }} \sim \sqrt{N}$, explaining why the aggregation is dominated by the translation mode in Fig. 6. On the other hand, when the cluster size $N$ is fixed and $X$ is increased, the shortest time scale for the translation mode is ultimately determined by clusters diffusion $t_{\text {trans }}^{\text {diff }} \sim X^{2} \ll t_{\text {trans }}^{\text {at }} \sim X \exp (X)$. The $X$ dependence of the Ostwald mode time scale $t_{\text {Ost }}^{\text {(diff) }}(X) \sim X^{2}$ is also quadratic. However, for large clusters $N \gg 1$, we have $t_{\text {Ost }}^{\text {(diff) }}=t_{\text {trans }}^{\text {(diff) }} / \sqrt{N}$, which explains the dominance of the Ostwald mode at large separation distances as shown in Fig. 8.

Finally, by examining the dependence on the attraction strength $\alpha$, we conclude that the Ostwald mode remains effectively frozen for strong attraction, because the time scale associated with the escape from the cluster increases exponentially with $\alpha$, i.e., $t_{\mathrm{Ost}}^{\mathrm{esc}}(\alpha) \sim e^{\alpha}$. The translation mode, on the other hand, increases its dominance, $t_{\text {trans }}^{\text {at }} \sim \alpha^{-1}$, due to increased attraction between the clusters. This linear increase of the translation mode eigenvalue with $\alpha$ is demonstrated by thick dotted line in Fig. 6(a).

We note that the qualitative estimates derived above cannot be used to describe the evaporation-condensation process in the case when the edge-to-edge separation distance between two clusters is comparable to or smaller than the cluster size. As a consequence, the stabilization of the Ostwald mode is not captured by the approximation.

\section{CONCLUDING REMARKS}

Phase separation occurs in order to minimize the total free energy of the system. A quenched initially uniform system forms clusters that then tend to merge to form bigger aggregates, because this reduces the amount of interfaces between phases and therefore the interfacial contribution to the free energy. The dynamics of the aggregation process, also known as coarsening dynamics, crucially depends on the distance between the clusters, their size, and the attraction strength. We have studied the coalescence of two equally sized clusters separated by a distance larger than the cluster size. The clusters can merge either as a result of their motion and subsequent collision, or as a result of the Ostwald ripening process, whereby particles diffuse from one cluster to another. This process is somewhat akin to an evaporation-condensation process, i.e., the particles evaporate from the smaller drop and then travel to condense on the larger drop.

We have shown that both coarsening modes can be suppressed and, consequently, the clustering can be "frozen" by a sufficiently strong pinning action of the external potential $U(x)$, associated with the corrugation of the channel walls. In the case of the smooth channel walls, we have demonstrated that the translation mode dominates the aggregation process for large cluster sizes and for strongly attracting particles. In fact, the Ostwald mode can be effectively arrested in the strong attraction limit. Finally, we have demonstrated that there is a crossover between the coarsening modes, induced by changing the separation distance between the clusters. Regardless of how strong the attraction is between the particles, the time scale associated with the translation mode can be rendered much larger than the Ostwald mode time scale by increasing the distance between clusters.

Although our results are for a simple 1D model, we believe that our overall conclusions about the behavior and the interplay of the two different coarsening modes should apply much more generally. We expect two-dimensional clusters aggregating on surfaces to behave in a qualitatively similar manner. In this situation, the corrugation potential $U(x)$ in our system models a rough potential due to the surface, with the parameter $\chi$ characterizing the surface roughness. Similarly, we expect our results to also be relevant to coarsening of clusters in three dimensions. To relate our results to these systems, one should think of the density profiles we calculate as corresponding to the cross-sectional density profile through a pair of two- or three-dimensional clusters. In fact, as mentioned above, because in one dimension there is no true phase transition, but in two and three dimensions there is a phase transition, the effect of fluctuations that to some extent have been neglected in the present mean-field treatment will be less influential in two and three dimensions. 
[1] I. M. Lifshitz and V. V. Slyozov, J. Phys. Chem. Solids 19, 35 (1961).

[2] C. Wagner, Z. Elektrochem. 65, 581 (1961).

[3] A. Onuki, Phase Transition Dynamics (Cambridge University Press, New York, 2002).

[4] R. C. Desai and R. Kapral, Dynamics of Self-Organised and SelfAssembled Structures (Cambridge University Press, Cambridge, 2009).

[5] J. A. Marqusee and J. Ross, J. Chem. Phys. 80, 536 (1984).

[6] P. Voorhees, J. Stat. Phys. 38, 231 (1985).

[7] J. H. Yao, K. R. Elder, H. Guo, and M. Grant, Phys. Rev. B 47, 14110 (1993).

[8] A. J. Bray, Adv. Phys. 43, 357 (1994).

[9] P. Meakin, Phys. Rev. Lett. 51, 1119 (1983).

[10] M. Kolb, R. Botet, and R. Jullien, Phys. Rev. Lett. 51, 1123 (1983).

[11] D. A. Weitz and M. Oliveria, Phys. Rev. Lett. 52, 1433 (1984).

[12] M. Y. Lin, H. M. Lindsay, D. A. Weitz, R. C. Ball, R. Klein, and P. Meakin, Nature (London) 339, 360 (1989).

[13] R. Botet and R. Jullien, Phys. Rev. Lett. 55, 1943 (1985).

[14] M. Filoche and B. Sapoval, Phys. Rev. Lett. 85, 5118 (2000).

[15] S. Großkinsky, M. Timme, and B. Naundorf, Phys. Rev. Lett. 88, 245501 (2002).

[16] C. Lutz, M. Kollmann, and C. Bechinger, Phys. Rev. Lett. 93, 026001 (2004).

[17] U. Thiele, L. Brusch, M. Bestehorn, and M. Bär, Eur. Phys. J. E 11, 255 (2003).

[18] K. B. Glasner and T. P. Witelski, Phys. Rev. E 67, 016302 (2003).

[19] L. M. Pismen and Y. Pomeau, Phys. Fluids 16, 2604 (2004).

[20] K. B. Glasner and T. P. Witelski, Physica D 209, 80 (2005).

[21] U. Thiele, in Thin Films of Soft Matter, edited by S. Kalliadasis and U. Thiele (Springer, Wien, 2007), pp. 25-93.

[22] V. Manoharan, M. T. Elsesser, and D. J. Pine, Science 301, 483 (2003).
[23] A. Stradner, H. Sedgwick, F. Cardinaux, W. C. K. Poon, S. U. Egelhaaf, and P. Schurtenberger, Nature (London) 432, 492 (2004).

[24] P. N. Segre, V. Prasad, A. B. Schofield, and D. A. Weitz, Phys. Rev. Lett. 86, 6042 (2001).

[25] A. I. Campbell, V. J. Anderson, J. S. van Duijneveldt, and P. Bartlett, Phys. Rev. Lett. 94, 208301 (2005).

[26] K. A. Dawson, Curr. Opin. Colloid Interf. Sci. 7, 218 (2002).

[27] U. M. B. Marconi and P. Tarazona, J. Chem. Phys. 110, 8032 (1999).

[28] U. M. B. Marconi and P. Tarazona, J. Phys.: Condens. Matter 12, A413 (2000).

[29] A. J. Archer and R. Evans, J. Chem. Phys. 121, 4246 (2004).

[30] A. J. Archer and M. Rauscher, J. Phys. A 37, 9325 (2004).

[31] R. Evans, Adv. Phys. 28, 143 (1979).

[32] R. Evans, Fundamentals of Inhomogeneous Fluids (Dekker, New York, 1992).

[33] J.-P. Hansen and I. R. McDonald, Theory of Simple Liquids, 4th ed. (Academic, London, 2013).

[34] J. K. Percus, J. Stat. Phys. 15, 505 (1976).

[35] A. Pototsky, A. J. Archer, M. Bestehorn, D. Merkt, S. Savel'ev, and F. Marchesoni, Phys. Rev. E 82, 030401(R) (2010).

[36] A. Pototsky, A. J. Archer, S. E. Savel'ev, U. Thiele, and F. Marchesoni, Phys. Rev. E 83, 061401 (2011).

[37] E. Doedel, H. B. Keller, and J. P. Kernevez, Int. J. Bifurcation Chaos 1, 493 (1991).

[38] Y. A. Kuznetsov, Elements of Applied Bifurcation Theory, 3rd ed. (Springer, New York, 2010).

[39] H. A. Dijkstra, F. W. Wubs, A. K. Cliffe, E. Doedel, I. F. Dragomirescu, B. Eckhardt, A. Y. Gelfgat, A. L. Hazel, V. Lucarini, A. G. Salinger, E. T. Phipps, J. Sanchez-Umbria, H. Schuttelaars, L. S. Tuckerman, and U. Thiele, Commun. Comput. Phys. 15, 1 (2014).

[40] E. Doedel, R. Paffenroth, A. R. Champneys, T. F. Fairgrieve, Y. A. Kuznetsov, B. Sandstede, and X. Wang, Technical Report, Caltech (2001), http://cmvl.cs.concordia.ca/auto/. 